\title{
RETRACTED ARTICLE: Determination of Dynamic Fracture Parameters of Westerly Granite Using the Notched Semi-circular Bend Technique in Split Hopkinson Pressure Bar Testing
}

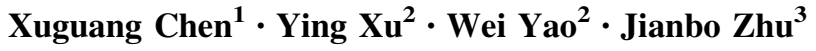

Received: 26 August 2016/Accepted: 29 December 2016/Published online: 17 January 2017

(c) Springer-Verlag Wien 2017

This technical note has been retracted by the authors under invitation of the editor in chief. Following a detailed note from a reader, pointing out substantial duplicating parts with previous articles published in other journals by the same authors, a thorough analysis of the published material was undertaken, involving the authors in the process. The conclusion reached is that this article is to be considered as a case of over-publication. While it is acceptable to repeat some information on methodology and test equipment, this should be limited. There is nothing wrong with publishing results on different rock materials, but the manuscripts should be significantly different and innovative. The online version of this article contains the full text of the retracted article as electronic supplementary material.

Electronic supplementary material The online version of this article (doi:10.1007/s00603-016-1163-1) contains supplementary material, which is available to authorized users.

\section{Wei Yao}

weiyao_84@163.com

1 College of Engineering, Ocean University of China,

Shandong Provincial Key Laboratory of Ocean Engineering, Qingdao 266100, China

2 State Key Laboratory of Hydraulic Engineering Simulation and Safety, School of Civil Engineering, Tianjin University, Tianjin 300072, China

3 Department of Civil and Environmental Engineering, The Hong Kong Polytechnic University, Hung Hom, Kowloon, Hong Kong 